PROCEEDINGS OF THE

AMERICAN MATHEMATICAL SOCIETY

Volume 131, Number 8, Pages 2337-2346

S 0002-9939(02)06816-8

Article electronically published on November 14, 2002

\title{
SOME FINITENESS CONDITIONS ON THE SET OF OVERRINGS OF AN INTEGRAL DOMAIN
}

\author{
ROBERT GILMER
}

(Communicated by Wolmer V. Vasconcelos)

\begin{abstract}
Let $D$ be an integral domain with quotient field $K$ and integral closure $\bar{D}$. An overring of $D$ is a subring of $K$ containing $D$, and $\mathcal{O}(D)$ denotes the set of overrings of $D$. We consider primarily two finiteness conditions on $\mathcal{O}(D)$ : $(\mathrm{FO})$, which states that $\mathcal{O}(D)$ is finite, and $(\mathrm{FC})$, the condition that each chain of distinct elements of $\mathcal{O}(D)$ is finite. (FO) is strictly stronger than (FC), but if $D=\bar{D}$, each of (FO) and (FC) is equivalent to the condition that $D$ is a Prüfer domain with finite prime spectrum. In general $D$ satisfies (FC) iff $\bar{D}$ satisfies (FC) and all chains of subrings of $\bar{D}$ containing $D$ have finite length. The corresponding statement for (FO) is also valid.
\end{abstract}

\section{INTRODUCTION}

All rings considered in this paper are assumed to be commutative and to contain an identity element. If $R$ is a subring of the ring $S$, we assume that $R$ and $S$ have the same identity element.

Suppose $D$ is an integral domain with quotient field $K$. In their study of residually algebraic pairs of integral domains in [2], Ayache and Jaballah encountered the following two conditions on the set of overring ${ }^{1}$ of $D$; we label these as (FO) and $(\mathrm{FC})$ :

(FO) $D$ has only finitely many overrings.

(FC) Each chain of distinct overrings of $D$ is finite.

These are the two main finiteness conditions referred to in the title of this paper. We use the term FO-domain or FC-domain to refer to an integral domain satisfying the respective condition (FO) or (FC). In extending results of [2] in [13] and [14, Jaballah asked [14, Quest. 1] for a characterization of FO-domains. We provide such characterizations in Theorems 3.1 and 3.4 of this paper, and a characterization of FC-domains is given in Theorem 2.14

It is clear that FO-domains are FC-domains. The converse fails in general, but an integrally closed FC-domain has only finitely many overrings. In fact, a characterization of integrally closed FC-domains can be easily obtained from results

Received by the editors January 15, 2002 and, in revised form, March 27, 2002.

2000 Mathematics Subject Classification. Primary 13G05, 13B02, 13B22, 13 F05.

Key words and phrases. Integral domain, overring, finite chains of overrings, finite prime spectrum, Prüfer domain.

${ }^{1}$ Recall that an overring of $D$ is a subring of $K$ containing $D$.

(C)2002 American Mathematical Society 
already in the literature. Theorem 1.5 shows that the class of integrally closed FCdomains coincides with the class of Prüfer domains with finite spectra.

To discuss our results in the case of non-integrally closed domains we introduce additional notations and terminology. If $R$ is a subring of $S$, we denote by $[R, S]$ the set of subrings of $S$ that contain $R$. Elements of $[R, S]$ are called $S$-overrings of $R$. In the case where $S$ is the total quotient ring of $R$, an $S$-overring of $R$ is called simply an overring of $R$ and we sometimes use the notation $\mathcal{O}(R)$ instead of $[R, S]$.

\section{BAsiC RESUlts AND THE INTEGRALly ClOSED CASE}

In this section we establish some basic properties of domains satisfying one of the conditions (FO) or (FC), and in Theorem 1.5 we characterize integrally closed domains satisfying these conditions.

Let $D$ be an integral domain with quotient field $K$ and denote by $\mathcal{O}(D)$ the set of overrings of $D$. It is clear that (FO) implies $(\mathrm{FC})$ and that $(\mathrm{FC})$ is equivalent to the validity of both a.c.c. and d.c.c. in $\mathcal{O}(D)$. In general the descending chain condition on intermediate rings of an extension $R \subseteq S$ implies that $S$ is a $P$-extension of $R$ in the terminology of Gilmer and Hoffman 11. B By definition this means that each element $s \in S$ is a root of a polynomial $f_{s}(t) \in R[t]$ such that at least one of the coefficients of $f_{s}(t)$ is a unit of $R$. We state this result formally:

Proposition 1.1. Suppose $R$ is a subring of the ring $S$. If d.c.c. is satisfied in $[R, S]$, then $S$ is a P-extension of $R$.

Proof. If $s \in S$, the decreasing chain $R[s] \supseteq R\left[s^{2}\right] \supseteq R\left[s^{4}\right] \supseteq \ldots$ in $[R, S]$ stabilizes, so $s^{2^{k}} \in R\left[s^{2^{k+1}}\right]$ for some $k$. Thus $s$ is a root of a polynomial $f(t)$ over $R$ such that the coefficient of $t^{2^{k}}$ in $f(t)$ is -1 , and hence $S$ is a $P$-extension of $R$.

Theorem 5 of [1] shows that for a domain $D$, the quotient field of $D$ is a $P$ extension of $D$ iff the integral closure of $D$ is a Prüfer domain. Proposition 1.1 therefore has the following corollary.

Corollary 1.2. Let $D$ be an integral domain with quotient field $K$. If d.c.c. is satisfied in $\mathcal{O}(D)$, the integral closure of $D$ is a Prüfer domain. In particular the integral closure of an FC-domain is a Prüfer domain.

If $R$ is a subring of $S$, we say that $S$ is strongly affine over $R$ (see [10]) if each $S$-overring of $R$ is finitely generated as a ring extension of $R$ (that is, is affine over $R$ ). By a familiar argument, a.c.c. is satisfied in $[R, S]$ iff $S$ is strongly affine over $R$. Thus we have:

Proposition 1.3. Suppose $D$ is a domain with quotient field $K$. If $D$ is an $F C$ domain, then $K$ is strongly affine over $D$; hence the integral closure of $D$ is a finite D-module.

Kaplansky [15, Sect. 1-3] uses the term $G$-domain 3 for a domain $D$ whose quotient field is an affine extension of $D$. An equivalent form of the definition is that the intersection of the set of nonzero prime ideals of $D$ (which is referred to as the pseudoradical of $D$ in [7]) is nonzero. A domain with finite spectrum is a

\footnotetext{
${ }^{2}$ In [6, p. 216], the more desirable terminology primitive extension is used for the concept. But because of several citations of results in [11, we continue to use the term $P$-extension here.

${ }^{3}$ The $G$ refers to (Oscar) Goldman, who considered domains of this type in [12].
} 
$G$-domain. The converse fails in general, but an FC-domain does, in fact, have finite spectrum. This assertion is a corollary to Theorem 1.5. The proof of (1.5) uses the following lemma, whose content is well-known.

Lemma 1.4. If $\left\{P_{i}\right\}_{i=1}^{n+1}$ is a set of pairwise incomparable prime ideals of the domain $D$, then $\bigcap_{i=1}^{n+1} D_{P_{i}}<\bigcap_{i=1}^{n} D_{P_{i}}$.

Theorem 1.5. Let $D$ be an integrally closed domain with quotient field $K$. The following conditions are equivalent:

(1) $D$ is a Prüfer domain with finite spectrum.

(2) $D$ is a finite-dimensional Prüfer domain with finite maximal spectrum.

(3) $D$ is an FO-domain.

(4) $D$ is an FC-domain.

Proof. The equivalence of (1) and (2) is well-known and the implication $(3) \Rightarrow(4)$ is patent. If (1) holds, then each overring of $D$ is an intersection of localizations $D_{P}$ of $D$ [8, (26.1)] and because $\operatorname{Spec}(D)$ is finite, the set of localizations of $D$ is also finite. Therefore $\mathcal{O}(D)$ is finite and (3) follows from (1). We show that (4) implies (2). Thus suppose that $D$ is an FC-domain. Corollary 1.2 shows that $D$ is a Prüfer domain, and Lemma 1.4 shows that $\operatorname{MSpec}(D)$ is finite. If $M \in \operatorname{MSpec}(D)$, then $D_{M}$ is an FC-domain and thus $M$ has finite height. Therefore $\operatorname{dim}(D)=$ $\sup \{\operatorname{ht}(M) \mid M \in \operatorname{MSpec}(D)\}$ is finite, and this establishes (2).

Corollary 1.6. An FC-domain has finite spectrum.

We note that if $D$ is assumed to be a Prüfer domain in the statement of Theorem 1.5. then that result is the same as Corollary 2.1 of [14]. In this regard, see also Remark 3.3

While (FC) implies (FO) in an integrally closed domain, this is not true in general. Using a classical $(D+M)$-construction, this can be made to depend upon the fact that in a finite-dimensional purely inseparable extension of fields, there may exist infinitely many intermediate fields.

Example 1.7. Let $F$ be a field of characteristic $p \neq 0$, let $X$ and $Y$ be indeterminates over $F$, let $K=F(X, Y)$, and let $L=F\left(X^{1 / p}, Y^{1 / p}\right)$. If $M$ is the maximal ideal of the domain $V=L[[t]]$ and if $J$ is the domain $K+M$, then $[J, V]$ is infinite because the extension $K<L$ has infinitely many intermediate fields. Therefore $J$ is not an FO-domain. But because each overring of $J$ is known to compare with $V$ under inclusion [8, Exer. 10, p. 271], each chain of distinct elements of $\mathcal{O}(J)$ has length at most 2, so $J$ is an FC-domain. (Note that Example 1.7 is essentially the same as Remark (b), page 610, of [1].)

\section{FC-Domains. Main Results}

Let $D$ be an integral domain with quotient field $K$ and integral closure $\bar{D}$. Our purpose in this section is to determine equivalent conditions for $D$ to be an FCdomain (see Theorem 2.14). This we do by first showing in Theorem 2.3 that $D$ is an FC-domain iff a.c.c. and d.c.c. hold in both $[D, \bar{D}]$ and $[\bar{D}, K]$. Then in Theorem 2.14 we show that these conditions are equivalent to (1)-(3): (1) $\bar{D}$ is a Prüfer domain with finite spectrum; (2) $\bar{D}$ is a finite $D$-module; (3) $D / C$ is an Artinian ring, where $C$ is the conductor of $D$ in $\bar{D}$. 
Our proof of Theorem [2.3 uses Proposition 2.2, and the proof of Proposition 2.2 uses, in turn, a variant of what is usually known as the $u, u^{-1}$ Lemma (see Theorem 67 of [15] or Lemma 19.14 of [8]). We state this variant below as Lemma [2.1] No proof of Lemma 2.1 is included here because the proof of (19.14) in [8] establishes Lemma 2.1 in a straightforward manner.

Lemma 2.1. Suppose $J$ is a subring of the quasilocal domain $(E, M)$, that $J$ is integrally closed in $E$, and that $u$ is a unit of $E$ which satisfies a polynomial in $J[t]$, one of whose coefficients is a unit of $J$. Then $u$ or $u^{-1}$ belongs to $J_{Q}$, where $Q=M \cap J$.

Proposition 2.2. Under the notation and hypotheses of Lemma 2.1 if $E$ is a $P$-extension of $J$, then $E=J_{Q}$.

Proof. The inclusion $J_{Q} \subseteq E$ is clear. For the other inclusion take $x \in E$. If $x \notin M$, then Lemma 2.1 shows that $x$ or $x^{-1}$ is in $J_{P}$. However, because $M$ lies over $P J_{P}$ in $J_{P}$ and neither $x$ nor $x^{-1}$ is in $M$, it follows that both $x$ and $x^{-1}$ are in $J_{P}$. If $x \in M$, then $1+x$ is a unit of $E$ and the proof just given implies that $1+x \in J_{P}$, so $x \in J_{P}$. In any case $x \in J_{P}$. Therefore $E \subseteq J_{P}$ and equality holds: $E=J_{P}$.

Theorem 2.3. Let $D$ be an integral domain with quotient field $K$ and integral closure $\bar{D}$. Then $D$ is an $F C$-domain iff a.c.c. and d.c.c. hold in both $[D, \bar{D}]$ and $[\bar{D}, K]$.

Proof. It is clear that a.c.c. and d.c.c. hold in $[D, \bar{D}]$ and in $[\bar{D}, K]$ if they hold in $[D, K]$. We prove the converse. Thus, assume first that both chain conditions hold in $[D, \bar{D}]$ and in $[\bar{D}, K]$ and that there exists an infinite strictly ascending sequence $E_{1}<E_{2}<\ldots$ of overrings of $D$. We seek a contradiction. Using bars to denote integral closure, $\bar{E}_{1} \subseteq \bar{E}_{2} \subseteq \ldots$ is an ascending sequence of overrings of $\bar{D}$, so $\bar{E}_{k}=\bar{E}_{k+1}=\ldots$ for some $\bar{k}$. It follows that each $E_{k+i}$ is integral over $E_{k}$ and each maximal ideal of $E_{k+i}$ is contracted from $E_{k+i+1}$ for each $i$. Therefore $\left|\operatorname{MSpec}\left(E_{k}\right)\right| \leq\left|\operatorname{MSpec}\left(E_{k+1}\right)\right| \leq \ldots \leq|\operatorname{MSpec}(\bar{D})|$, with the last inequality holding because, by Theorem $1.5 \bar{D}$ is a Prüfer domain with finite spectrum and $\bar{E}_{k}$ is an overring of $\bar{D}$. Therefore there exists $m \geq k$ such that $\left|\operatorname{MSpec}\left(E_{m}\right)\right|=\left|\operatorname{MSpec}\left(E_{m+1}\right)\right|=\ldots=v$. Without loss of generality we assume that $m=1, \operatorname{MSpec}\left(E_{i}\right)=\left\{M_{i, j}\right\}_{j=1}^{v}$, and $M_{i+1, j}$ lies over $M_{i, j}$ in $E_{i}$ for all $i$ and $j$. Then $E_{i}=\bigcap_{j=1}^{v}\left(E_{i}\right)_{M_{i, j}}<E_{i+1}=\bigcap_{j=1}^{v}\left(E_{i+1}\right)_{M_{i+1, j}}$ and $\left(E_{i}\right)_{M_{i, j}} \subseteq\left(E_{i+1}\right)_{M_{i+1, j}}$ for all $i$ and $j$. Thus, for each $i$ there exists at least one $j$ (depending on $i$ ) such that the inclusion $\left(E_{i}\right)_{M_{i, j}} \subseteq\left(E_{i+1}\right)_{M_{i+1, j}}$ is proper. It follows that there exists a $j$ between 1 and $v$ such that the inclusion $\left(E_{i}\right)_{M_{i, j}} \subseteq\left(E_{i+1}\right)_{M_{i+1, j}}$ is proper for infinitely many values of $i$. Thus, there exists an infinite strictly ascending sequence $\left\{\left(F_{i}, Q_{i}\right)\right\}_{i=1}^{\infty}$ of quasilocal overrings of $D$ (and hence of $D_{M}$, where $M$ is a maximal ideal of $D$ containing $Q_{1} \cap D$ ), where $Q_{i+1} \cap F_{i}=Q_{i}$ for each $i$. We show that this leads to a contradiction. Let $J_{i}=F_{i} \cap \bar{D}$ and let $P_{i}=Q_{i} \cap \bar{D}$. If $x \in F_{i}$ is integral over $J_{i}$, then $x$ is integral over $D$ and hence $x \in \bar{D} \cap F_{i}=J_{i}$. Therefore $J_{i}$ is integrally closed in $F_{i}$. Since $\bar{D}$ is a Prüfer domain, $K$ is a $P$-extension of $D$, and in particular $F_{i}$ is a $P$-extension of $J_{i}$. We have shown that the hypotheses of Proposition 2.2 are satisfied for the extension $J_{i} \subseteq F_{i}$, so that the result shows that $F_{i}=\left(J_{i}\right)_{P_{i}}$ for each $i$. Because a.c.c. holds in $[D, \bar{D}]$, there exists $q$ such that $J_{q}=J_{q+1}=\ldots$ Moreover, $P_{q} \subseteq P_{q+1} \subseteq \ldots$ and finite-dimensionality of $J_{q}$ 
implies that $P_{t}=P_{t+1}=\ldots$ for some $t \geq q$, and this implies that $F_{t}=F_{t+1}$, a contradiction. We conclude that a.c.c. holds in $[D, K]$.

The proof that d.c.c. holds in $[D, K]$ has many similarities with the proof just given and we abbreviate details. Thus, if there exists an infinite strictly decreasing sequence $E_{1}>E_{2}>\ldots$ of overrings of $D$, then for some $k, \bar{E}_{k}=\bar{E}_{k+1}=\ldots$ and $\left|\operatorname{MSpec}\left(E_{k}\right)\right|=\left|\operatorname{MSpec}\left(E_{k+1}\right)\right|=\ldots$. Again this leads to the fact that there exists an infinite strictly decreasing sequence $\left\{\left(F_{i}, Q_{i}\right)\right\}_{i=1}^{\infty}$ of quasilocal overrings of $D$, where $Q_{i} \cap F_{i+1}=Q_{i+1}$ for each $i$. Letting $J_{i}=F_{i} \cap \bar{D}$ and $P_{i}=Q_{i} \cap \bar{D}$, d.c.c. in $[D, \bar{D}]$ implies that there exists $q$ such that $J_{q}=J_{q+1}=\ldots$. Again Proposition 2.2 implies that $F_{i}=\left(J_{i}\right)_{P_{i}}$ for each $i$, where $P_{q} \supseteq P_{q+1} \supseteq \ldots$. Finite-dimensionality of $J_{q}$ implies $P_{t}=P_{t+1}$ for some $t \geq q$, and this leads to the contradiction that $F_{t}=F_{t+1}$. Therefore d.c.c. also holds in $[D, K]$ and the proof is complete.

We remark that the proof of Theorem 2.3 shows that (FC) is a local property. Note that Corollary 1.6 shows that the hypothesis of finite maximal spectrum is necessary in the statement of Theorem 2.4 .

Theorem 2.4. Suppose $D$ is a domain with finite maximal spectrum. Then $D$ is an $F C$-domain iff $D_{M}$ is an $F C$-domain for each maximal ideal $M$ of $D$.

In light of the characterization of integrally closed FC-domains in Theorem 1.5 Theorem 2.3 reduces the general characterization problem to that of determining conditions under which a.c.c. and d.c.c. hold in $[D, \bar{D}]$. To resolve this case, we use some results concerning minimal extensions, where the definition is as follows. If $R$ is a proper subring of the ring $S$, we say that $S$ is a minimal extension of $R$ (or that $R<S$ is a minimal extension) if there exists no proper intermediate ring, that is, if $|[R, S]|=2$. Minimal extensions arise in various contexts throughout the literature. Here we cite results Lemma 2.5 from [9] and Propositions 2.6 and 2.7 from the (unpublished) doctoral dissertations [16] and 3]. We then prove Theorem 2.8 , though parts of this result appear in the sources just named.

Lemma 2.5 ([9, Lemmas 2.1 and 2.2]). Suppose $R<S$ is a minimal extension and let $C$ be the conductor of $R$ in $S$. If $A$ and $B$ are ideals of $S$ such that $A \cap R=B \cap R$, then $A \cap R=A \cap B$, so $A \cap R$ is a subset of $C$. If $P \in \operatorname{Spec}(R)$, at most two prime ideals of $S$ lie over $P$ in $R$.

Note that if $R<S$ is a minimal extension and if $T$ is the integral closure of $R$ in $S$, then either $T=R$ or $T=S$. Thus either $R$ is integrally closed in $S$, or else $S$ is integral over $R$. In our context we are interested in the second of these two cases.

Proposition 2.6 ([16, Thm. 1 and Cor. 1]). Suppose $R<S$ is a minimal extension with conductor $C$, and assume that $S$ is integral over $R$.

(1) $C$ is a maximal ideal of $R$.

(2) If $P \in \operatorname{Spec}(R), P \nsubseteq C$, then $R_{P}=S_{(R-P)}$.

Proposition 2.7 (3, Cor. 2.8, Prop. 4.1]). Suppose $R<S$ is a minimal integral extension with conductor $C$. If $I$ is an ideal $R$ that is not contained in $C$, then $I$ is contracted from $S, I S$ in the unique ideal of $S$ lying over $I$ in $R$, and $S / I S \cong R / I$.

Theorem 2.8. Suppose $R<S$ is a minimal integral extension with conductor $C$. Then either (i) $C$ is maximal in $S$, (ii) there exists a maximal ideal $M$ of $S$ such that $M^{2} \subseteq C<M$, or (iii) there exists distinct maximal ideals $M_{1}, M_{2}$ of $S$ such that $C=M_{1} \cap M_{2}$. 
Proof. Suppose $C$ is not maximal in $S$. Since $S / R$ is integral, $C$ is contracted from a maximal ideal of $S$, and Lemma 2.5 shows that at most two maximal ideals of $S$ lie over $C$ in $R$; moreover, if $C$ is contracted from two maximal ideals of $S$, then (iii) is satisfied. Thus we need only show that if $M$ is the unique maximal ideal of $S$ lying over $C$, then $M^{2} \subseteq C$. If $J \neq M$ is an ideal of $S$ between $C$ and $M$, we must have $C=J \cap R$. Therefore $M \cap J=J=C$ by Lemma 2.5. Hence no ideal of $S$ lies properly beween $C$ and $M$, and this implies that $M N \subseteq C$ for some maximal ideal $N$ of $S$ [17, p. 237], [8, Exer. 11, Sect. 38]. If $N \neq M$, then $C+N=S$ since $\operatorname{rad}(C)=M$, and hence $M=M C+M N \subseteq C$, and this contradiction shows that $M=N$. Therefore $M^{2} \subseteq C$, as we wished to show.

Minimal extensions are relevant in the consideration of a.c.c. and d.c.c. in $[D, \bar{D}]$ because these two chain conditions imply that there exists a chain $D=D_{0}<D_{1}<$ $\ldots<D_{n}=\bar{D}$ of intermediate rings, where the extension $D_{i}<D_{i+1}$ is minimal for each $i$.

Our next goal is to show that if $D$ is an (FC)-domain and if $C$ is the conductor of $D$ in $\bar{D}$, then $D / C$ is an Artinian ring (see Theorem 2.12). Our proof uses some preliminary results.

Lemma 2.9. If $M$ is a maximal ideal of the ring $R$ and if $R / M^{2}$ is Artinian, then $R / M^{n}$ is Artinian for each positive integer $n$.

Proof. Clearly $R / M^{n}$ is zero-dimensional, so it suffices to show that $R / M^{n}$ is Noetherian. By Cohen's Theorem it suffices to show that $M / M^{n}$ is finitely generated. Since $M / M^{2}$ is finitely generated, $M=I+M^{2}$ for some finitely generated ideal $I$ of $R$. In $R / I$ we have $(M / I)=\left(M^{2}+I\right) / I=(M / I)^{2}$, and hence $M / I=(M / I)^{n}=\left(M^{n}+I\right) / I$. It follows that $M=M^{n}+I$ and $M / M^{n}$ is finitely generated.

We remark that the hypothesis in Lemma 2.9 that $M$ is maximal is not necessary for the conclusion that $R / M^{n}$ is Artinian. That is, if $I$ is an ideal of $R$ such that $R / I^{2}$ is Artinian, then $R / I^{n}$ is Artinian for each $n \in \mathbb{Z}$. To see this it again suffices to show that $R / I^{n}$ is Noetherian. This follows from an inductive argument, based solely upon the assumption that $R / I^{2}$ is Noetherian. Thus, the proof of Lemma 2.9 shows that $I / I^{2}$ finitely generated implies that $I / I^{k}$ is finitely generated for each positive integer $k$, and it is known [8, Exer. $10(3), \S 40]$ that $R / A B$ is a Noetherian ring if $R / A$ and $R / B$ are Noetherian and $A / A B$ is a finitely generated $R$-module. It is not true, of course, that $R / J^{2}$ is Noetherian (or Artinian) if $R / J$ has the same property, and such examples exist with $J$ a maximal ideal of $R$.

Lemma 2.10. Let $M$ be a maximal ideal of a semiquasilocal Prüfer domain $D$. Then $D / M^{n}$ is Artinian for each positive integer $n$.

Proof. By Lemma 2.9 it suffices to show that $M / M^{2}$ is finitely generated. If $M=$ $M^{2}$, this is clear. If $M \neq M^{2}$, choose $x \in M, x \notin M^{2} \cup\left(\bigcup_{i=1}^{v} M_{i}\right)$, where $\left\{M_{i}\right\}_{i=1}^{v}$ is the set of maximal ideals of $D$ distinct from $M$. Since $M^{2}$ is $M$ primary, $M D_{M}>M^{2} D_{M}$ and $M D_{M}$ is principal, generated by any element of $M D_{M}-M^{2} D_{M}$. In particular, $M D_{M}=x D_{M}$, and because $M D_{M_{i}}=D_{M_{i}}=x D_{M_{i}}$ for each $i$, it follows that $M=x D$. Hence $M / M^{2}$ is principal.

Lemma 2.11. Suppose $D<E$ is a minimal extension of integral domains. If $E$ is a finite $D$-module and if $E / M^{2}$ is Artinian for each maximal ideal $M$ of $E$, then $D / P^{2}$ is Artinian for each maximal ideal $P$ of $D$. 
Proof. Let $P$ be a maximal ideal of $D$ and let $C$ be the conductor of $D$ in $E$. If $P \neq C$, then Proposition 2.6] shows that no power of $P$ is contained $C$. Moreover, Proposition 2.7] shows that $P E$ is maximal in $E$ and $E /(P E)^{n} \cong D / P^{n}$ for each $n$. Hence $D / P^{2}$ is Artinian by hypothesis. If $P=C$, then Proposition 2.6 shows that either (i) $P$ is maximal in $E$, (ii) $P=M N$, where $M$ and $N$ are distinct maximal ideals of $E$, or (iii) there exists a maximal ideal $M$ of $E$ such that $M^{2} \subseteq P<M$. In case (i), $D / P^{2}$ is a subring of the Artinian ring $E / P^{2}$, a finite $D / P^{2}$-module. By the theorem of Eakin-Nagata (see [5]), $D / P^{2}$ is Noetherian, hence Artinian. A similar argument applies in case (ii); there $D / P^{2}=D / M^{2} N^{2}$ is a subring of the Artinian ring $E / M^{2} N^{2} \cong E / M^{2} \oplus E / N^{2}$, where $E / M^{2} N^{2}$ is a finite $\left(D / M^{2} N^{2}\right)$ module. In case (iii), the argument of case (i) shows that the ring $D / M^{4}$ is Artinian since, by Lemma2.9, $E / M^{4}$ is Artinian. Consequently, $D / P^{2} \cong\left(D / M^{4}\right) /\left(P^{2} / M^{4}\right)$ is also an Artinian ring.

Theorem 2.12. Suppose $D$ is an integral domain with integral closure $\bar{D}$, a Prüfer domain with finite spectrum. Let $C$ be the conductor of $D$ in $\bar{D}$. If a.c.c. and d.c.c. hold in $[D, \bar{D}]$, then $D / C$ is an Artinian ring.

Proof. Since a.c.c and d.c.c. hold in $[D, \bar{D}]$, there exists an ascending sequence $D=D_{0}<D_{1}<D_{2}<\ldots<D_{m}=\bar{D}$ of intermediate rings such that $D_{i}<D_{i+1}$ is a minimal extension for $0 \leq i \leq m-1$. Moreover, a.c.c. in $[D, \bar{D}]$ implies that $D_{i+1}$ is a finite $D_{i}$-module for each $i$. Results $2.9-2.11$ then imply that $D / P^{n}$ is an Artinian ring for each maximal ideal $P$ of $D$ and each positive integer $n$. It follows that $D / P_{1} P_{2} \cdots P_{s}$ is Artinian for any (not necessarily distinct) maximal ideals $P_{1}, P_{2}, \ldots, P_{s}$ of $D$. Let $C_{i}$ be the conductor of $D_{i}$ in $D_{i+1}$ for $0 \leq i \leq m-1$. Proposition 2.6 shows that $C_{i}$ is a maximal ideal of $D_{i}$, and since $D_{i}$ is integral over $D, C_{i} \cap D=Q_{i}$ is maximal in $D$. We observe that $C_{0} C_{1} \cdots C_{s-1}$ is an ideal of $\bar{D}$ that is contained in $D$. Therefore $C \supseteq C_{0} C_{1} \cdots C_{s-1} \supseteq Q_{0} Q_{1} \cdots Q_{s-1}$, and because $D / Q_{0} Q_{1} \cdots Q_{s-1}$ is Artinian, its homomorphic image $D / C$ is also Artinian.

Proposition 2.13. Suppose $D$ is an integral domain with integral closure $\bar{D}$ and let $C$ be the conductor of $D$ in $\bar{D}$. If $\bar{D}$ is a finite $D$-module and if $D / C$ is an Artinian ring, then both a.c.c. and d.c.c. hold in $[D, \bar{D}]$.

Proof. It suffices to show that both a.c.c. and d.c.c. hold in $[D / C, \bar{D} / C]$, but this is clear from the hypothesis: $D / C$ is an Artinian ring and $\bar{D} / C$ is a finite $D / C$-module, so $\bar{D} / C$ is both Artinian and Noetherian as a $D / C$-module.

Theorem 2.14. Let $D$ be an integral domain with integral closure $\bar{D}$ and let $C$ be the conductor of $D$ in $\bar{D}$. Then $D$ is an FC-domain if and only if the following three conditions are satisfied:

(1) $\bar{D}$ is a Prüfer domain with finite spectrum.

(2) $\bar{D}$ is a finite $D$-module.

(3) $D / C$ is an Artinian ring.

Proof. If $D$ is an FC-domain, then Proposition [1.3, Theorem 1.5] and Theorem 2.12 show that (1)-(3) are satisfied. And conversely, if (1)-(3) hold, then $D$ is an FC-domain by Theorems 2.3, 1.5, 2.12 and Proposition 2.13,

For a domain $D$ with finite maximal spectrum, Theorem 2.4 shows that the joint conditions a.c.c. and d.c.c. hold in $\mathcal{O}(D)$ iff they hold in $\mathcal{O}\left(D_{M}\right)$ for each $M \in \operatorname{MSpec}(D)$. It is interesting to note that the corresponding equivalences are 
also valid for the conditions a.c.c. and d.c.c. considered separately. We state this result as Proposition 2.15 its proof is elementary.

Proposition 2.15. Suppose $D$ is an integral domain with finite maximal spectrum. Then a.c.c. holds in $\mathcal{O}(D)$ iff it holds in $\mathcal{O}\left(D_{M}\right)$ for each maximal ideal $M$ of $D$. The corresponding statement for d.c.c. is also valid.

Proof. We give the proof for a.c.c. If a.c.c. holds in $\mathcal{O}(D)$, it clearly holds in each $\mathcal{O}\left(D_{M}\right)$. Conversely, suppose $\operatorname{MSpec}(D)=\left\{M_{i}\right\}_{i=1}^{s}$, let $N_{i}=D-M_{i}$ for each $i$, and assume that a.c.c. holds in $\mathcal{O}\left(D_{M_{i}}\right)$ for each $i$. If $E_{1} \subseteq E_{2} \subseteq \ldots$ is an ascending chain of overrings of $D$, then $\left(E_{1}\right)_{N_{i}} \subseteq\left(E_{2}\right)_{N_{i}} \subseteq \ldots$ is an ascending chain of overrings of $D_{M_{i}}$ for $1 \leq i \leq s$. Thus, there exists an integer $k$ such that $\left(E_{k}\right)_{N_{i}}=\left(E_{k+1}\right)_{N_{i}}=\ldots$ for each $i \geq k$. Because $E_{j}=\bigcap_{i=1}^{s}\left(E_{j}\right)_{N_{i}}$, it follows that $E_{k}=E_{k+1}=\ldots$, and a.c.c. holds in $\mathcal{O}(D)$, as was to be proved.

\section{FO-DOMAINS}

In this section we characterize FO-domains (Theorems 3.1 and 3.4). Such domains have finite spectra since they have only finitely many localizations or, alternatively, because an FO-domain is an FC-domain. We begin by proving results that parallel, for (FO), Theorems 2.3 and 2.4 for (FC).

Theorem 3.1. Let $D$ be an integral domain with quotient field $K$ and integral closure $\bar{D}$. Then $D$ is an FO-domain iff each of the sets $[D, \bar{D}]$ and $[\bar{D}, K]$ is finite.

Proof. It is clear that the stated condition is necessary. For sufficiency, assume that $[D, \bar{D}]$ and $[\bar{D}, K]$ are finite. Theorem 1.5 shows that $\bar{D}$ is a Prüfer domain with finite spectrum, and hence $K$ is a $P$-extension of $D$. Since each domain is an intersection of quasilocal domains, to show that $\mathcal{O}(D)$ is finite, it suffices to show that it contains only finitely many quasilocal domains. Let $[D, \bar{D}]=\left\{J_{i}\right\}_{i=1}^{k}$. Each $J_{i}$ has finite spectrum since its integral closure $\bar{D}$ has finite spectrum. Hence the set $\mathcal{S}$ of domains that are localizations of some $J_{i}, 1 \leq i \leq k$, is finite. We show that $\mathcal{S}$ is the set of quasilocal overrings of $D$; the proof is included in the proof of Theorem 2.3 but we repeat it here for completeness. If $(E, M)$ is a quasilocal overring of $D$, let $J=E \cap \bar{D}$ and let $Q=M \cap \bar{D}=M \cap J$. Then $J$ is integrally closed in $E$ and $E$ is a $P$-extension of $J$, so Proposition 2.2 shows that $E=J_{Q} \in \mathcal{S}$. Thus the set of quasilocal overrings of $D$ is finite, and this completes the proof.

Theorem 3.2. Suppose $D$ is a domain with finite maximal spectrum. Then $D$ is an FO-domain iff $D_{M}$ is an FO-domain for each $M \in \operatorname{MSpec}(D)$.

Proof. Let $\operatorname{MSpec}(D)=\left\{M_{i}\right\}_{i=1}^{s}$. We need only prove that $\mathcal{O}(D)$ is finite if each $\mathcal{O}\left(D_{M_{i}}\right)$ is finite. Let $\mathcal{T}=\bigcup_{i=1}^{s} \mathcal{O}\left(D_{M_{i}}\right)$, a finite set. To show that $\mathcal{O}(D)$ is finite it suffices, as in the proof of Theorem 2.3 , to show that $D$ has only finitely many quasilocal overrings. Let $(E, M)$ be a quasilocal overring of $D$. If $Q=M \cap D$, then $Q$ is contained in some $M_{i}$ and $D_{M_{i}} \subseteq D_{Q} \subseteq E$. It follows that $E \in \mathcal{O}\left(D_{M_{i}}\right) \subseteq \mathcal{T}$, so $\mathcal{T}$ is the (finite) set of quasilocal overrings of $D$.

Remark 3.3. Anderson, Dobbs, and Mullins [1] have investigated finiteness of $[R, S]$ for a ring extension $R \subseteq S$. If $[R, S]$ is finite, they say that $R \subseteq S$ has FIP (for the "finitely many intermediate $R$-algebras" property). Thus " $D$ is an FO-domain" translates in their terminology to " $D \subseteq K$ has FIP", where $K$ is the quotient field of $D$. Theorem 2.6 of [1] is closely related to our Theorem 3.1 In fact, apart from 
an error in its statement, it would imply Theorem 3.1; the error in its statement is in the assertion that an FO-domain is an $i$-domain. For a counterexample, let $V_{1}$ and $V_{2}$ be distinct finite-dimensional valuation domains on a field $L$ and assume that $V_{i}=K+M_{i}$, where $K$ is a subfield of $L$ and $M_{i}$ is the maximal ideal of $V_{i}$. Let $D=K+\left(M_{1} \cap M_{2}\right)$. The integral closure of $D$ is $\bar{D}=V_{1} \cap V_{2}$, a Prüfer domain with finite spectrum, and since $\bar{D} /\left(M_{1} \cap M_{2}\right) \cong K \oplus K$ is a minimal extension of $D /\left(M_{1} \cap M_{2}\right) \cong K, \bar{D}$ is a minimal extension of $D$. Therefore $D$ is an FO-domain by Theorems 3.1 and 1.5. $D$ is not an $i$-domain since, for example, $M_{1} \cap \bar{D}$ and $M_{2} \cap \bar{D}$ have the same contraction to $D$. If the base domain (denoted $R$ ) in Theorem 2.6 of [1] is assumed to be integrally closed, then there is no problem with the proof. Hence this result (as well as Proposition 2.8(b)(c) of [1], which cites Theorem 2.6 in its proof) includes our Theorem 1.5

As used in the preceding remark, it is true in general that if $R$ is a subring of the ring $S$ and $I$ is a common ideal of $R$ and $S$, then $R \subseteq S$ has FIP iff $R / I \subseteq S / I$ has FIP. Thus we have:

Theorem 3.4. Let $D$ be an integral domain with integral closure $\bar{D}$ and let $C$ be the conductor of $D$ in $\bar{D}$. Then $D$ is an FO-domain iff $\bar{D}$ is a Prüfer domain with finite spectrum and the extension $D / C \subseteq \bar{D} / C$ has $F I P$.

A more definitive form of the condition that $D / C \subseteq \bar{D} / C$ has FIP would be welcome. In light of Theorem 2.14 such a form might be anticipated, for that result shows that for an FO-domain $D, D / C$ and $\bar{D} / C$ are Artinian. Because $\bar{D}$ is a Prüfer domain, $\bar{D} / C$ is, in fact, a PIR. Also, Theorem 3.2 shows that we may pass to the case where $D$ and $D / C$ are quasilocal. This is a topic that appears to be worthy of further investigation. The characterization of FIP that is almost completely resolved in [1] is where the base ring is a field. Even there the characterization involves several cases; we cite Theorem 3.8 of [1]:

Theorem 3.5 ([1, Thm. 3.8]). Let $K$ be a field. For a ring extension $K \subseteq T$, consider the following four conditions:

(1) $K$ is finite and $T$ is a finite-dimensional $K$-vector space.

(2) $K$ is infinite, $T$ is a reduced ring, and $T=K[\alpha]$ for some $\alpha \in T$ which is algebraic over $K$.

(3) $K$ is infinite and $T=K[\alpha]$ for some $\alpha \in T$ such that $\alpha^{3}=0$.

(4) $K$ is infinite and $T=K[\beta]=K[\alpha] \times K_{2} \times \cdots \times K_{n}$, where $\beta$ is algebraic over $K, \alpha \in T$ satisfies $\alpha^{3}=0$, and for each $i=2, \ldots, n, K \subseteq K_{i}$ is a field extension which has FIP.

Then:

(a) If $K \subseteq T$ has FIP, then at least one of the conditions (1)-(4) holds.

(b) If at least one of the conditions (1)-(3) holds, then $K \subseteq T$ has FIP.

(c) If $K$ is a perfect field and condition (4) holds, then $K \subseteq T$ has FIP.

(d) Assume further that $K$ is a perfect field. Then $K \subseteq T$ has FIP iff at least one of the conditions (1)-(4) holds,

In seeking to extend Theorem 3.5 to, say, the case where $R$ is local Artinian and $T$ is a PIR, the characterization will involve more than the extension of the residue

\footnotetext{
${ }^{4}$ The integral domain $D$ is an $i$-domain if the canonical map $\operatorname{Spec}(E) \rightarrow \operatorname{Spec}(D)$ is an injection for each overring $E$ of $D$.
} 
field of $R$ by the residue fields of $T$ (and hence more than one or more applications of Theorem 3.5). This is indicated by Example 3.6

Example 3.6. Let $n_{1}<\ldots<n_{s}$ be a finite set of positive integers with greatest common divisor 1 . Let $K$ be a field and let $D$ be the power series ring $K\left[\left[t^{n_{1}}, \ldots, t^{n_{s}}\right]\right]$, where $t$ is an analytic indeterminate over $K$. The integral closure of $D$ is $\bar{D}=K[[t]]$, a DVR which is a finitely generated $D$-module. The conductor of $D$ in $\bar{D}$ is $C=t^{m} \bar{D}$, where $m$ is the smallest positive integer such that all integers $u \geq m$ belong to the submonoid of $\mathbb{Z}_{0}$ generated by $\left\{n_{i}\right\}_{i=1}^{s}$. The ring $D / C$ is Artinian, so Theorem 2.14 shows that $D$ is an FC-domain, whatever the choice of $n_{1}, n_{2}, \ldots, n_{s}$. Lemma 3.6 of [1] shows, however, that $D$ may or may not be an FO-domain. For example, if $K$ is infinite, then $\left.K\left[t^{3}, t^{4}, t^{5}\right]\right]$ is an FOdomain, but $K\left[\left[t^{4}, t^{5}, t^{6}, t^{7}\right]\right]$ is not. Note that $D$ and $\bar{D}$, and hence $D / C$ and $\bar{D} / C$, have the same residue field $K$ for all possible choices of $\left\{n_{i}\right\}_{1}^{s}$.

\section{REFERENCES}

1. D.D. Anderson, D. Dobbs, and B. Mullins, The primitive element theorem for commutative algebras, Houston J. Math., 25 (1999), 603-623.

2. A. Ayache and A. Jaballah, Residually algebraic pairs of rings, Math. Zeit., 225 (1997), 49-65. MR 98d:13004

3. L. Dechéne, Adjacent Extensions of Rings, Ph.D. Dissertation, University of California at Riverside, 1978.

4. D.E. Dobbs, On chains of overrings of an integral domains, Internat. J. Comm. Rings (to appear).

5. P. Eakin, The converse to a well-known theorem on Noetherian rings, Math. Ann., 177 (1968), 278-282. MR 37:1360

6. M. Fontana, J. A. Huckaba, and I. Papick, Prüfer Domains, Marcel Dekker, New York, 1997. MR 98d:13021

7. R. Gilmer, The pseudo-radical of a commutative ring, Pacific J. Math., 19 (1966), 275-284. MR 34:4294

8. R. Gilmer, Multiplicative Ideal Theory, Queen's Papers Pure Appl. Math., Volume 90, Queen's University, Kingston, 1992. MR 93j:13001

9. R. Gilmer and W. Heinzer, Intersections of quotient rings of an integral domain, J. Math. Kyoto Univ., 7 (1967), 133-150. MR 36:6397

10. R. Gilmer and W. Heinzer, Finitely generated intermediate rings, J. Pure Appl. Algebra, 37 (1985), 237-264. MR 86m:13006

11. R. Gilmer and J. Hoffmann, A characterization of Prüfer domains in terms of polynomials, Pacific J. Math., 60 (1975), 81-85. MR 54:302

12. O. Goldman, Hilbert rings and Hilbert's Nullstellensatz, Math. Zeit., 54 (1951), 136-140. MR 13:427d

13. A. Jaballah, A lower bound for the number of intermediary rings, Comm. in Algebra, 27 (1999), 1307-1311. MR 99k:13028

14. A. Jaballah, Finiteness of the set of intermediary rings in normal pairs, Saitama Math. J., 17 (1999), 59-61. MR 2000j:13012

15. I. Kaplansky, Commutative Rings, Allyn \& Bacon, Boston, 1970. MR 40:7234

16. M.L. Modica, Maximal Subrings, Ph.D. Dissertation, University of Chicago, 1975.

17. O. Zariski and P. Samuel, Commutative Algebra, Vol. I, Springer, New York, 1975. MR 52:5641

Department of Mathematics, Florida State University, Tallahassee, Florida 323064510

E-mail address: gilmer@math.fsu.edu 